\begin{tabular}{|c|c|}
\hline Title & Emoticon recommendation system for effective communication \\
\hline Author(s) & Urabe, Y uki; Rzepka, Rafal; A raki, Kenji \\
\hline Citation & $\begin{array}{l}\text { A dvances in Social Networks A naly sis and Mining 2013, A SONA M '13, Niagara, ON, Canada- A ugust } 25 \text { - 29, } 2013 \text {, } \\
\text { 1460-1461 } \\
\text { https://doi.org/10.1145/2492517.2492594 }\end{array}$ \\
\hline Issue Date & $2013-08$ \\
\hline Doc URL & http://hdl.handle.net/2115/63966 \\
\hline Rights & $\begin{array}{l}\text { (c) } 2013 \text { IEEE. Personal use of this material is permitted. Permission from IEEE must be obtained for all other uses, in } \\
\text { any current or future media, including reprinting/republishing this material for advertising or promotional purposes, } \\
\text { creating new collective works, for resale or redistribution to servers or lists, or reuse of any copyrighted component of } \\
\text { this work in other works. }\end{array}$ \\
\hline Tyре & proceedings (author version) \\
\hline File Information & Emoticon recommendation system for effective communication.pdf \\
\hline
\end{tabular}

Instructions for use 


\title{
Emoticon Recommendation System for Effective Communication
}

\author{
Yuki Urabe, Rafal Rzepka, and Kenji Araki \\ Graduate School of Information Science and Technology, \\ Hokkaido University \\ Sapporo, 060-0814, Japan \\ \{y_urabe, kabura, araki\}@media.eng.hokudai.ac.jp
}

\begin{abstract}
The existence of social media has made computermediated communication more widespread among users around the world. This paper describes the development of an emoticon recommendation system that allows users to express their feelings with their input. In order to develop this system, an innovative emoticon database consisting of a table of emoticons with points expressed from each of 10 distinctive emotions was constructed. An evaluation experiment showed that $71.3 \%$ of user-selected emoticons were among the top 10 emoticons recommended by the proposed system.
\end{abstract}

Keywords-emoticon recommendation, recommendation method, affect sensing and analysis, text processing.

\section{INTRODUCTION}

Today, the social networks Facebook ${ }^{1}$ and Twitter $^{2}$ handle 665 million $^{3}$ and 288 million $^{4}$ active users respectively throughout the world. Such services have dramatically increased social user interaction on the Internet from the days when only e-mail and online chatting systems existed. However, due to a lack of nonverbal cues such as facial expressions, body movements, and emotional tones, computermediated communication (CMC) often fails to present personal dispositions that are transparently expressed in face-to-face communication. Hence, users compensate for these shortcomings by using emoticons.

This paper presents an Emoticon Recommendation System (ERS) that recommends appropriate emoticons to the user input sentence. The system is comprised of an emoticon database which we developed for this system and an affect analysis system, ML-Ask [1], which was previously developed in our laboratory. The system recommends Eastern-type emoticons (e.g. "( $\left.\wedge_{-} \wedge\right)$ ", in contrast to Western-type emoticons, ":-)"), which are mainly used in Japan. Moreover, Eastern-type emoticons have a great number of emoticons recorded in an online emoticon dictionary $(58,130$ emoticons are recorded in an Eastern emoticon dictionary ${ }^{5}$ while a Western emoticon dictionary ${ }^{6}$ records 260 emoticons). Therefore, we consider

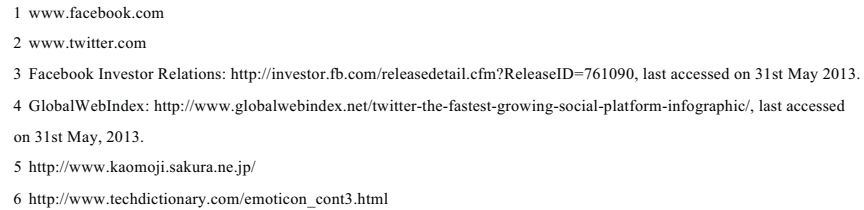

Eastern-type emoticons sophisticated enough to express users' feelings in CMC.

\section{EMOTICON RECOMMENDATION SYSTEM}

The system utilizes two main procedures (Fig. 1). First, the system analyzes the emotion in the user input. We used an affect analysis system: ML-Ask [1]. Secondly, the system rearranges the emoticon database in the order of suitability to the emotion specified by ML-Ask and recommends the top five emoticons to the user. The original emoticon database was created by performing a survey of Japanese university students. The system continues to recommend the next five emoticons until the user chooses one for the sentence. Lastly, the system outputs the user input with the chosen emoticon.

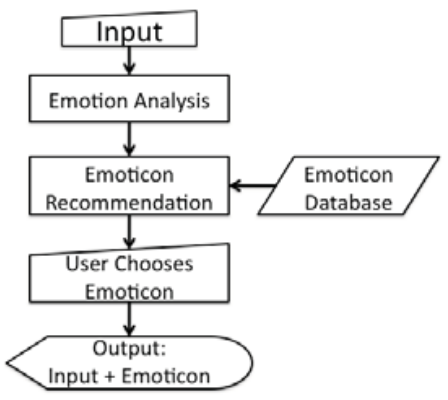

Fig. 1. System Procedure

A. ML-Ask

ML-Ask [1] was previously developed by Ptaszynski et al. in our laboratory. ML-Ask separates emotive utterances from non-emotive utterances and specifies the specific emotion types in the emotive utterances. It uses 10 distinctive emotion types as defined by Nakamura [2]. These are: "joy/delight", "anger", "excitement", "sadness/gloom", "liking/fondness", "fear", "relief", "dislike", "surprise/amazement", and "shyness". ERS utilizes the result of the emotion types obtained from ML-Ask and reorders the emoticon database.

\section{B. Emoticon Database}

The original emoticon database was developed by adopting an idea of Kawakami's [3]. The database is organized in a table of 59 emoticons with the points expressed from each of 10 
distinctive emotions [2]. These points (Fig. 2) are an average of ratings given by 60 Japanese university students (Fig. 3).

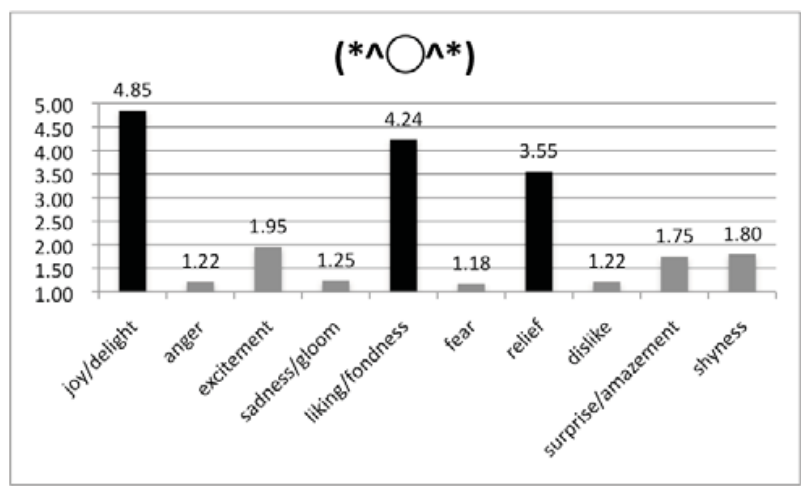

Fig. 2. Example of average rated values for an emoticon

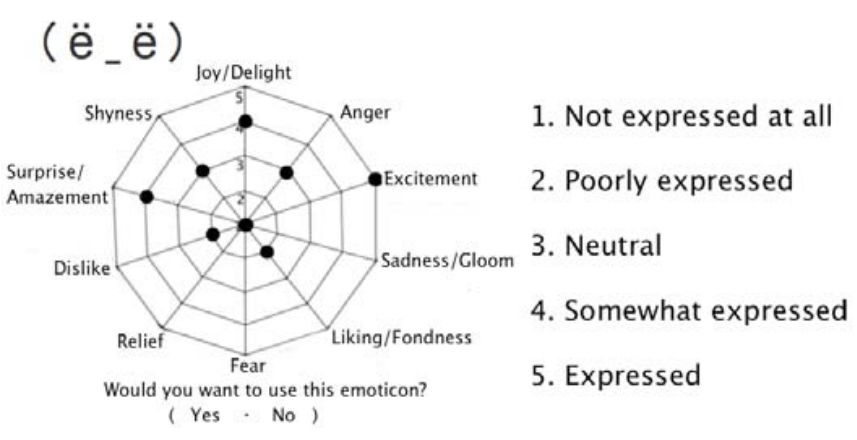

Fig. 3. Example of emoticon ratings in each of the 10 emotions

\section{EVALUATION EXPERIMENT}

Comparative experiments were performed to confirm that the proposed emoticon recommendation system is superior to the current system, which recommends emoticons from users' chosen emoticons in the past. The current system can be seen on the iPhone ${ }^{7}$ 's Japanese keyboard. We independently developed a system using the same method as the current system used on the iPhone in order to coordinate the proposed system and the interface. The experiment was performed for 10 days from $22^{\text {nd }}$ Oct, 2012 to $31^{\text {st }}$ Oct, 2012 with the cooperation of 20 Japanese undergraduate and masters students, consisting of 8 men and 12 women.

The participants were given a list of 15 emotive words (in Japanese) and asked to create and input sentences for each word and evaluate each system. We employed the semantic differential scale [4] for the evaluation of the system.

\section{DISCUSSION AND CONCLUSIONS}

We examined the emoticons chosen by participants from both systems. Fig. 4 shows the percentage of emoticons that were chosen among the top 10 in the emoticon database (the database is reordered into each emotion type according to the result from ML-Ask.). We can see a significant difference between the two systems: we achieved $71.3 \%$ on average with the proposed system, while we only achieved $45.0 \%$ on average with the current system. From this result, we can reasonably state that people are motivated to choose emoticons to express their feelings, and the proposed system can assist them in choosing more appropriate emoticons than the current system. Moreover, we can consider that participants may procrastinate when choosing emoticons, meaning that they tend to choose an emoticon in the higher-listed options rather than carefully looking for the most appropriate one in the list. The current system may be useful for people who choose a limited variety of emoticons; however, the system will become more useful, enabling users to choose appropriate emoticons easily if we integrate the current system and the proposed system.

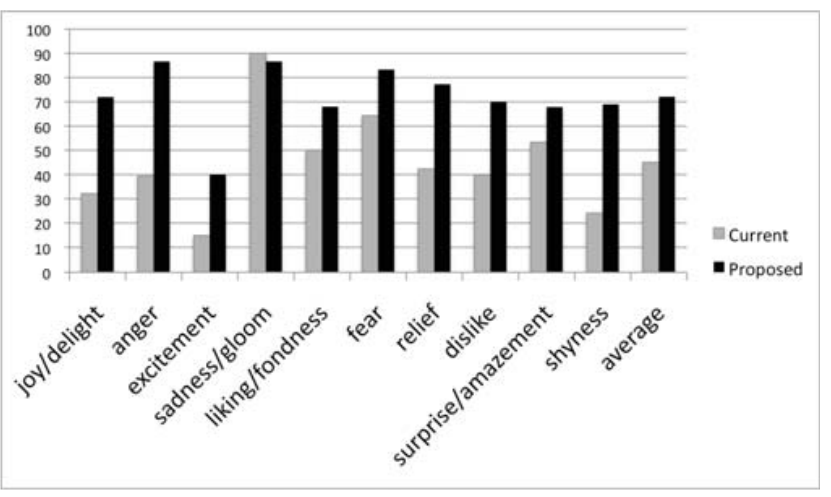

Fig. 4. The percentage of chosen emoticons ranked top 10 in the database

Our future work will be to integrate both systems in order to make the system more useful to a wide range of users. Moreover, expansion of the emoticon database is required. More emoticons in the database will be helpful for discovering the types of symbols that articulate each emotion type and creating a system to generate emoticons fitting to the user input. Also, this emoticon database will be helpful for sentiment analysis not only in social media, but also in all computermediated content.

At present, the system only works on terminal emulators such as Terminal in Mac OS $\mathrm{X}^{8}$. However, because smartphone users are significantly increasing in recent years, we will develop an application for smartphone users and investigate how the system appeals to users. Moreover, we plan to make the proposed method open source for developers, so that anyone can build in any kind of communicative interface.

\section{REFERENCES}

[1] M. Ptaszynski, D. Pawel, R. Rzepka, and K. Araki, "Effective analysis of emotiveness in utterances based on features of lexical and non-lexical layer of speech", Proceedings of the Fourteenth Annual Meeting of the Association for Natural Language Processing, pp. 171-171, 2008.

[2] A. Nakamura, "Dictionary of Emotive Expressions (Kanjyo Hyogen Jiten)", Tokyodo Publishing, 1993.

[3] M. Kawakami, "The database of 31 Japanese emoticon with their emotions and emphasis.", The Human Science Research Bulletin of Osaka Shoin Women's University, vol. 7, pp. 67-82, 2008.

[4] C. E. Osgood, G. J. Suci, and P. H. Tannenbaum, "The measurement of meaning", University of Illinois Press, Urbana, USA, 1957. 